\title{
New Constructions in Axonometric System Fundamentals
}

\author{
Lázaro Gimena, Faustino N. Gimena and Pedro Gonzaga \\ Department of Projects Engineering, Public University of Navarre, Campus Arrosadia C.P. 31006, Pamplona, Navarre, Spain
}

\begin{abstract}
In this communication we start both from a trirectangular trihedral defined by the three coordinate planes and a fourth plane, called the chart or projection, which contains the vertex of the trihedral above. Later we define the main related views or trihedral views as those produced by the tilt of the three coordinate planes to the outside of their trihedral on the chart plane. For each projection plane there are some unique main related views, regardless of the projection direction. In turn, from the trihedral views we can obtain the axonometric perspective from which they come. These properties can simplify some graphic constructions of the axonometric system, for example, determine the projections of the axes from the main related views or determine the axonometric scales from the axes. It is considered that this new definition of related views allows a simplification as to the understanding of the representation systems and it is intended to be suitable for teaching purposes and programming.
\end{abstract}

Key words: Axonometric system, central projection, main related views.

\section{Introduction}

At present knowledge of the axonometric perspective is perfectly standardised and assumed, but this has not happened throughout history and it has taken many centuries to achieve the complete representation system formulation that concerns us [1]. Axonometric perspective is scientifically systematised since the mathematical intervention. In $1820 \mathrm{~W}$. Farish in his work, Treatise on isometrical perspective, he presents a new method of projection that is in fact an isometric orthogonal axonometry [2]. In 1844 L. J. Weisbach in his work, Die monodimetrische und axonometrische. Projections methode provides the mathematical basis of orthogonal axonometric perspective [3]. In the document Orthogonale Axonometrie of $1905 \mathrm{R}$. Schüssler systematises the orthogonal axonometric perspective [4]. With this system of representation it was clearly defined from an operational point of view, with its most frequent use in scientific and technical fields [5]. At present, there is little research on the axonometric perspective. It has

Corresponding author: Faustino N. Gimena, $\mathrm{PhD}$, research fields: engineering and architecture projects, analysis of structures formed by 3D-curved beam element, hydrological models of rainfall-runoff. E-mail: faustino@unavarra.es. been studied in depth perception [6] and the symbolic value [7] of this system of representation. It is also considered that the axonometric perspective helps describing the drawing plane and, usually, it is presented as a standard characteristic in CAD systems and other visual computation tools [8]. Descriptive geometry has been widespread using all the techniques of images of abstract objects and their relationships [9]. Research continues on the orthogonal and oblique axonometric [10]. In this paper we extend the knowledge of the axonometric perspective and we define Main Related Views as those produced by the tilt of the three coordinate planes to the outside of their trihedral on the main chart plane. We also get the axonometric perspective from these main related views. With this we intend to simplify some graphic constructions of the axonometric system in order to improve the transmission of its scientific content.

\section{New Axonometric Constructions}

Firstly orthogonal axonometric perspective fundamentals are presented to improve the graphic and constructive comprehension of the axonometric system, combining the graphic content with the analytic expression of the elements and new geometric 
operations used. Then new constructions are developed for the tilt of the three coordinate planes on the main chart plane and a new concept of related views is presented.

\subsection{Axonometric System Fundamentals}

We start from a trirectangular trihedral shown in (Fig. $1)$, called system trihedral or reference trihedral $(O)(x)(y)(z)$, formed by the coordinated planes $(x)(O)(y),(y)(O)(z)$ and $(z)(O)(x)$.

The three lines or edges, defined by the coordinate planes intersect at a point called vertex noted in the following way:

$$
(O)=[0,0,0]
$$

On each of the lines and from the vertex we carry a magnitude unit, which establishes in each of the lines $(x),(y)$ and $(z)$ the points $(I),(J)$ and $(K)$ respectively.

This points' coordinates are:

$$
(I)=[u, 0,0],(J)=[0, u, 0] \text { and }(K)=[0,0, u]
$$

A fourth plane called $\Pi$ is set as a projection plane or main chart plane, it passes by the vertex.

Its normalised equation is:

$$
\begin{aligned}
& \text { where } \sqrt{a_{x}{ }^{2}+a_{y}{ }^{2}+a_{z}^{2}}=u
\end{aligned}
$$

The orthogonal projection direction $p_{\Pi}$ on that plane is:

$$
p_{\Pi}=\left[a_{x}, a_{y}, a_{z}\right]
$$

Trying to achieve the analytical expression of the system elements we consider that the reference trihedral is immovable. The only one able to change its position is the main chart plane but always passing through the point of origin. If we project orthogonally the system trihedral on the main chart plane, we will achieve the axonometric perspective formed by the lines $x, y$ and $z$, intersecting at the vertex $O \equiv(O)$.

The points $(I),(J)$ and $(K)$ can be projected in a similar way, getting respectively points $I, J$ and $K$.

These are the coordinates of those projected unitary points of the system trihedral:

$$
\begin{aligned}
& I=\left[u^{2}-a_{x}{ }^{2},-a_{x} a_{y},-a_{x} a_{z}\right] / u \\
& J=\left[-a_{y} a_{x}, u^{2}-a_{y}{ }^{2},-a_{y} a_{z}\right] / u \\
& K=\left[-a_{z} a_{x},-a_{z} a_{y}, u^{2}-a_{z}{ }^{2}\right] / u
\end{aligned}
$$

The magnitudes $\overline{O I}=u_{x}, \overline{O J}=u_{y}$ and $\overline{O K}=u_{z}$ are called orthogonal axonometric scales. From the magnitudes above it is possible to conclude these relationships:

$$
u_{x}=\sqrt{u^{2}-a_{x}^{2}}, u_{y}=\sqrt{u^{2}-a_{y}^{2}}, u_{z}=\sqrt{u^{2}-a_{z}^{2}}
$$

where $u_{x}^{2}+u_{y}^{2}+u_{z}^{2}=2 u^{2}$

$$
\begin{aligned}
& a_{x}=\sqrt{\left(u_{y}{ }^{2}+u_{z}{ }^{2}-u_{x}{ }^{2}\right) / 2} \\
& a_{y}=\sqrt{\left(u_{x}{ }^{2}+u_{z}{ }^{2}-u_{y}{ }^{2}\right) / 2} \\
& a_{z}=\sqrt{\left(u_{x}{ }^{2}+u_{y}{ }^{2}-u_{z}{ }^{2}\right) / 2}
\end{aligned}
$$

Fig. 1 also shows the angles formed between the axes $x, y$ and $z$ in the axonometric perspective whose values are:

$$
\begin{aligned}
& \text { FOJ }=\alpha_{z}=\operatorname{acos}\left(-a_{x} a_{y} / u_{x} u_{y}\right) \\
& \text { FOK }=\alpha_{x}=\operatorname{acos}\left(-a_{y} a_{z} / u_{y} u_{z}\right) \\
& \text { KOI }=\alpha_{y}=\operatorname{acos}\left(-a_{x} a_{z} / u_{x} u_{z}\right)
\end{aligned}
$$

Furthermore, from the figure it is concluded that:

$$
\alpha_{x}+\alpha_{y}+\alpha_{z}=2 \pi
$$

Projections of the reference trihedral axes can take many different angles, leading to many types of orthogonal axonometric perspectives: isometric (3 equal angles), diametric (only 2 angles are the same) and trimetric (3 different angles).

Fig. 1 shows the projection of the system trihedral on the main chart plane. Each intersection line $c_{z}, c_{x}$ and $\mathrm{c}_{\mathrm{y}}$ is perpendicular both to $(z),(x)$ and $(y)$ and to their projections $z, x$ and $y$ respectively. This lines directions can be noted in this way:

$$
\begin{aligned}
& c_{z}=\Pi \cap(x)(O)(y) \equiv\left[-a_{y}, a_{x}, 0\right] \\
& c_{x}=\Pi \cap(y)(O)(z) \equiv\left[0,-a_{z}, a_{y}\right] \\
& c_{y}=\Pi \cap(z)(O)(x) \equiv\left[a_{z}, 0,-a_{x}\right]
\end{aligned}
$$

Up to this point minimal elements underlying orthogonal axonometric system have been expressed. They are needed to understand the operations presented in this article. 


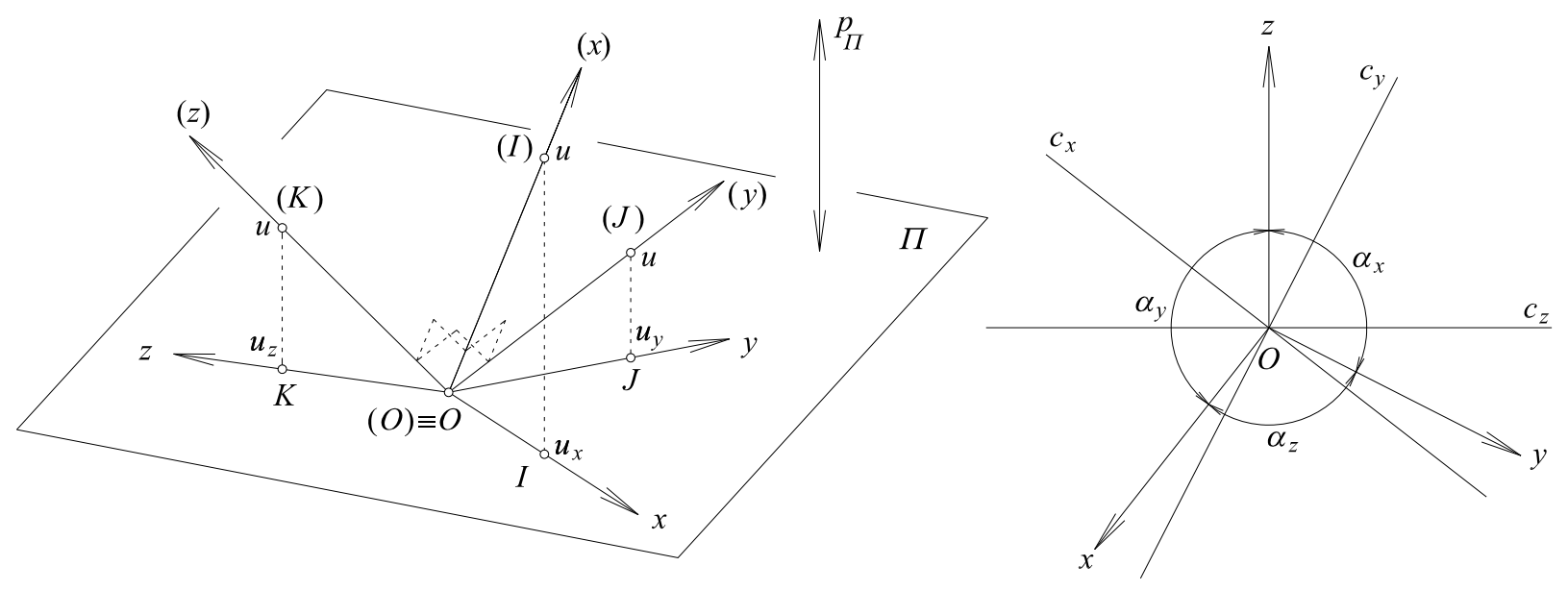

Fig. 1 System elements and trihedral system intersection with the main chart plane.

\subsection{A Coordinate Plane Tilted Down on the Main Chart} Plane

The steps to get the tilt of a coordinate plane on the main chart plane are shown in Fig. 2.

$(x)(O)(y)$ plane is chosen to be tilted, by point $I$ of $x$-axis at distance $u_{x}$ from point $O$, we draw a line parallel to the $y$-axis.

The intersection of this line with the line $c_{z}$ determines the point $P_{z}$. The angle $\vartheta(I) P_{z}$ is straight, therefore three points $O,(I)$ and $P_{z}$ form a right triangle. This triangle is the tilted down one on the main chart plane. $c_{z}$ is used as hinge line.

To do this, we draw a circle whose diameter passes through the points $O$ and $P_{z}$, under a straight angle.

The intersection between this circle and the line through point $I$ that is perpendicular to hinge line $c_{z}$ (parallel to $z$ ) determines the tilted down point $(I)_{z}$.

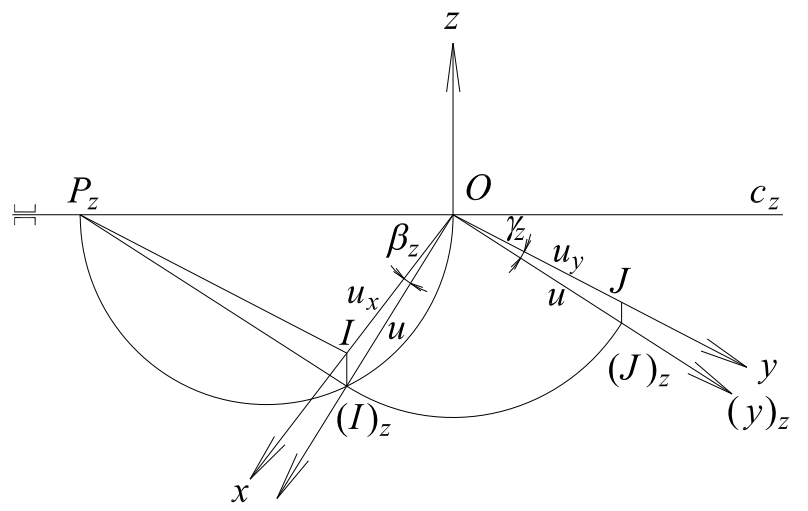

$(x)_{z}$

Fig. 2 Tilt of a coordinate plane on the main chart plane.
The distance $\overline{O(I)_{z}}$ measures a unit magnitude $u$ and its direction determines the tilted axis $(x)_{\mathrm{z}}$.

Through the point $O$ we draw a line $(y)_{z}$ perpendicular to $(x)_{\mathrm{z}}$ (parallel to $\overline{P_{z}(I)_{z}}$ line).

This two lines represent the tilt of the side $(x) O(y)$ of the system trihedral on the main chart plane $\Pi$. Within the coordinate plane $(x) O(y)$ to tilt, the same operations could have been done from point $J$ on $y$-axis, instead of point $I$ on the $x$-axis.

If we draw the unit magnitude $u$ from $O$ on the tilted line $(y)_{z}$, we will get point $(J)_{z}$.

The line through this point and perpendicular to the hinge line $c_{z}$ determines point $J$ on the $y$-axis. Distance $\overline{O J}$ is the magnitude $u_{y}$ on the $y$-axis.

\subsection{Trihedral Planes Tilt on the Main Chart Plane}

In Fig. 3 we continue developing the above procedure to show the tilt of the coordinate planes that form the reference system trihedral on the main chart plane. It can be seen that each edge of the system trihedral folds twice on the main chart plane.

This is because each edge is associated to two coordinate planes. It also can be seen that lines $\overline{I(I)_{z}}$ and $\overline{J(J)_{z}}$ are parallel to line $\overline{O K}$ placed on the $z$-axis. The same thing happens with lines $\overline{I(I)_{y}}$ and $\overline{K(K)_{y}}$, in relation to line $\overline{O J}$ on $y$-axis and lines $\overline{J(J)_{x}}$ and $\overline{K(K)_{x}}$ to line $\overline{O I}$ placed on $x$-axis. 


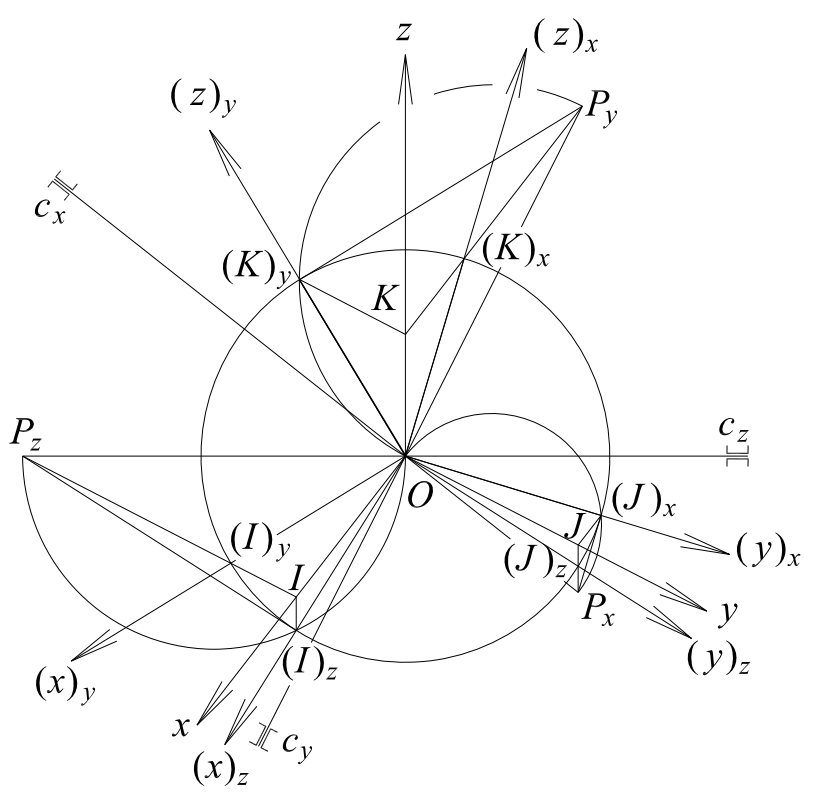

Fig. 3 Trihedral planes tilted on the main chart plane.

The six coordinates of the tilted unit points $(I),(J)$ and $(K)$ are:

In relation to plane $(x)(O)(y)$

$$
\begin{aligned}
& (I)_{z}=\left[a_{z}+a_{y}{ }^{2} /\left(u+a_{z}\right),-a_{x} a_{y} /\left(u+a_{z}\right),-a_{x}\right] \\
& (J)_{z}=\left[-a_{x} a_{y} /\left(u+a_{z}\right), a_{z}+a_{x}^{2} /\left(u+a_{z}\right),-a_{y}\right]
\end{aligned}
$$

In relation to plane $(y)(O)(z)$

$$
\begin{aligned}
& (J)_{x}=\left[-a_{y}, a_{x}+a_{z}^{2} /\left(u+a_{x}\right),-a_{y} a_{z} /\left(u+a_{x}\right)\right] \\
& (K)_{x}=\left[-a_{z},-a_{y} a_{z} /\left(u+a_{x}\right), a_{x}+a_{y}^{2} /\left(u+a_{x}\right)\right]
\end{aligned}
$$

In relation to plane $(z)(O)(x)$

$$
\begin{aligned}
& (K)_{y}=\left[-a_{x} a_{z} /\left(u+a_{y}\right),-a_{z}, a_{y}+a_{x}^{2} /\left(u+a_{y}\right)\right] \\
& (I)_{y}=\left[a_{y}+a_{z}^{2} /\left(u+a_{y}\right),-a_{x},-a_{x} a_{z} /\left(u+a_{y}\right)\right]
\end{aligned}
$$

Angles formed between the axonometric axis and the tilted axis are called and noted in the following way (Figs. 2-3):

$x$-axis:

$$
\begin{aligned}
& \digamma(I)_{z}=\beta_{z}=\operatorname{acos}\left(\left(a_{y}^{2} /\left(u+a_{z}\right)+a_{z}\right) / \sqrt{u^{2}-a_{x}^{2}}\right) \\
& \oiiint(I)_{y}=\gamma_{y}=\operatorname{acos}\left(\left(a_{z}^{2} /\left(u+a_{y}\right)+a_{y}\right) / \sqrt{u^{2}-a_{x}^{2}}\right)
\end{aligned}
$$

y-axis:

$$
\begin{aligned}
& \mathscr{W}(J)_{x}=\beta_{x}=\operatorname{acos}\left(\left(a_{z}^{2} /\left(u+a_{x}\right)+a_{x}\right) / \sqrt{u^{2}-a_{y}^{2}}\right) \\
& \mathscr{( O}(J)_{z}=\gamma_{z}=\operatorname{acos}\left(\left(a_{x}^{2} /\left(u+a_{z}\right)+a_{z}\right) / \sqrt{u^{2}-a_{y}^{2}}\right)
\end{aligned}
$$

z-axis:

$$
\begin{aligned}
& K O(K)_{y}=\beta_{y}=\operatorname{acos}\left(\left(a_{x}^{2} /\left(u+a_{y}\right)+a_{y}\right) / \sqrt{u^{2}-a_{z}^{2}}\right) \\
& K O(K)_{x}=\gamma_{x}=\operatorname{acos}\left(\left(a_{y}^{2} /\left(u+a_{x}\right)+a_{x}\right) / \sqrt{u^{2}-a_{z}^{2}}\right)
\end{aligned}
$$

From the Figs.1-3 are deduced the following equations between angles:

$$
\alpha_{x}=\pi / 2+\beta_{x}+\gamma_{x} ; \alpha_{y}=\pi / 2+\beta_{y}+\gamma_{y} ; \alpha_{z}=\pi / 2+\beta_{z}+\gamma_{z}
$$

Although there are a lot more relationships between axonometric system elements, we have only presented the ones needed for the understanding of this paper.

\section{Main Related Views}

It is known that the axonometric system is to represent figures in a plane, using cylindrical projection, referred to three orthogonal axes, so that they retain their proportions in the three directions of space: height, width and length.

Therefore, axonometry is the transfer between dihedral or related views of a piece and the perspective projection of a piece. Below, a new concept of main related views is presented

\subsection{Main Related Views of a Coordinate Plane}

Fig. 4 shows the steps to get, on the tilt of one of the coordinate planes of the reference trihedral system, the true shape of a plane figure contained in that plane in the perspective.

Taking point $L$ whose projections on the $x$ and $y$ axes are respectively the aforementioned points $I$ and $J$; points $O, I, J$ and $L$ form a unit square in the plane $(x)(O)(y)$.

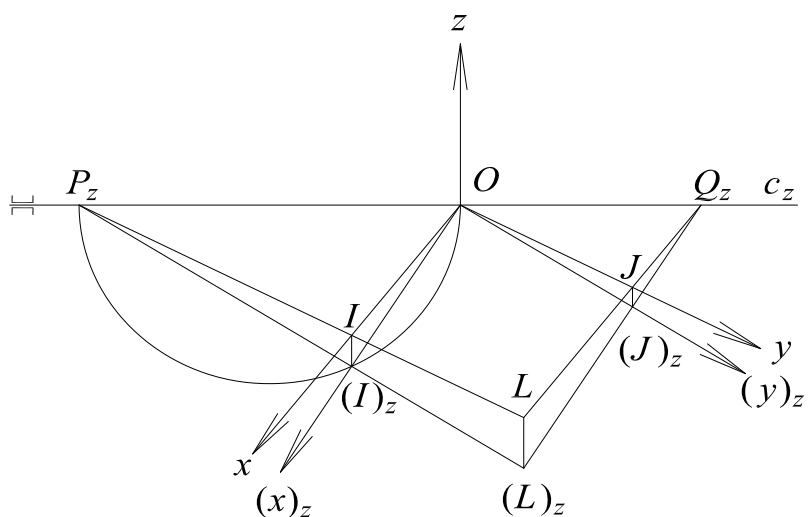

Fig. 4 Main related view of a coordinate plane. 
Given the affine connection between the aforementioned plane figure and its tilt, getting tilted point $(L)_{\mathrm{z}}$ is immediate taking hinge line $c_{z}$ as affinity axis, and the perpendicular direction to it as affinity direction. It should be noted that in that construction, in addition to origin $O$, points $P_{z}$ and $\mathrm{Q}_{\mathrm{z}}$ are double. Any other point in the plane in the perspective can be transported to the related view through these operations, and vice versa.

\subsection{Trihedral Planes' Main Related Views}

Following the method of previous section, it is possible to get the main related views associated to the three planes of the reference trihedral.

Fig. 5 shows point $D$, whose projections on the coordinate planes are points $L, M$ and $N$. Point $D$ is a vertex of a unit cube. $\overline{O D}$ is a diagonal and it is supported on the trihedral planes.

The unit edge squares which form the orthogonal views of the aforementioned cube are presented in each of the three main related views.

Those views are guided on the main chart plane according to the established affinities from traces $c_{z}, c_{y}$ and $c_{z}$.

In the same way, any point of the orthogonal guided views as well as main related views, can be equally transported to the perspective.

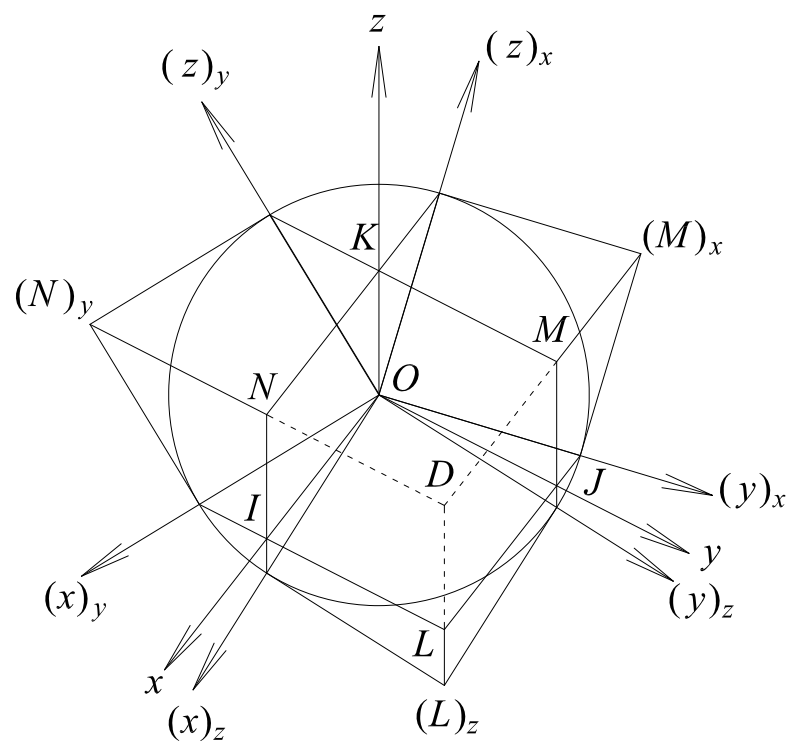

Fig. 5 Main related views.
In Fig. 6 main related views of a unit cube are shown as well as some elementary magnitudes associated with it.

\section{Axonometry Construction from Main Related Views}

A new concept of main related views has been already presented. In this section we intend to build the axonometric axes with its angles and scales from the existing relationship between these views.

\subsection{Main Related Views Position}

By guiding the orthogonal views of an object to represent according to Fig. 7, it is possible to interpret same as main related views of some trimetry.

The quadrants of the three tilted-down reference trihedral planes $(x)_{\mathrm{z}} \mathrm{O}(y)_{\mathrm{z}},(\mathrm{y})_{\mathrm{z}} \mathrm{O}(\mathrm{z})_{\mathrm{z}}$ and $(z)_{\mathrm{y}} O\left({ }_{\mathrm{x}}\right)_{\mathrm{y}}$ on the main chart plane $\Pi$ form the following related views position angles:

$$
\begin{aligned}
& \delta_{x}=\beta_{z}+\gamma_{y}=\operatorname{acos}\left(\frac{a_{y}{ }^{2}+a_{z}{ }^{2}+a_{y} a_{z}+u\left(a_{y}+a_{z}\right)}{\left(u+a_{y}\right)\left(u+a_{z}\right)}\right) \\
& \delta_{y}=\beta_{x}+\gamma_{z}=\operatorname{acos}\left(\frac{a_{z}{ }^{2}+a_{x}{ }^{2}+a_{z} a_{x}+u\left(a_{z}+a_{x}\right)}{\left(u+a_{z}\right)\left(u+a_{x}\right)}\right) \\
& \delta_{z}=\beta_{y}+\gamma_{x}=\operatorname{acos}\left(\frac{a_{x}{ }^{2}+a_{y}{ }^{2}+a_{x} a_{y}+u\left(a_{x}+a_{y}\right)}{\left(u+a_{x}\right)\left(u+a_{y}\right)}\right)
\end{aligned}
$$

By making some operations on Eq. (18), it is possible to get this simplified expression:

$$
\frac{1-\cos \delta_{x}}{a_{x}{ }^{2}\left(u+a_{x}\right)}=\frac{1-\cos \delta_{y}}{a_{y}{ }^{2}\left(u+a_{y}\right)}=\frac{1-\cos \delta_{z}}{a_{z}{ }^{2}\left(u+a_{z}\right)}
$$

Additionally from Eqs. (9) and (18) we get:

$$
\delta_{x}+\delta_{y}+\delta_{z}=\pi / 2
$$

Some geometric properties have been noted mathematically, deduced from the position between the main related views.

\subsection{Definition of Axonometry from the Main Related Views}

In order to define from the main related views the axonometric axes with its scales, and thus reconstruct the perspective of the object, we can proceed as follows. 


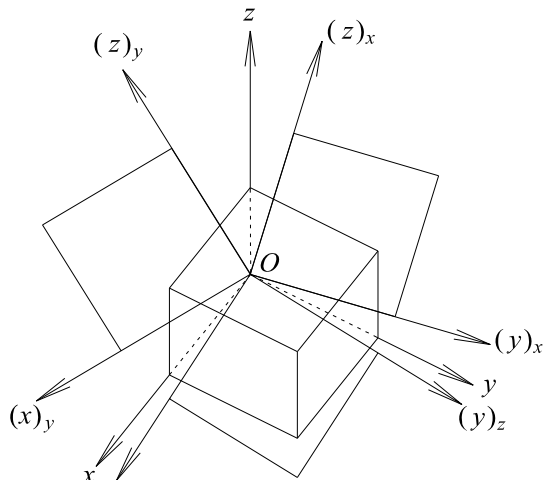

$(x)_{z}$

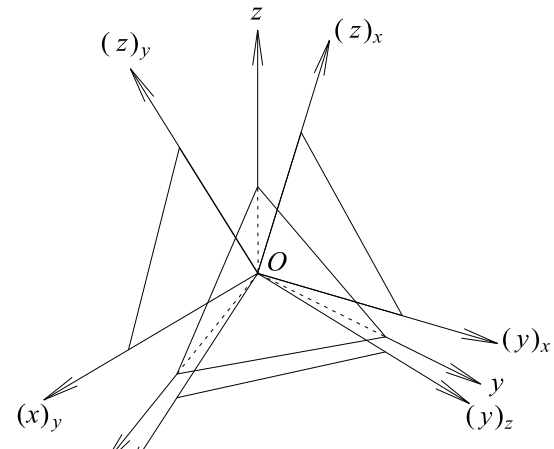

$(x)_{z}$

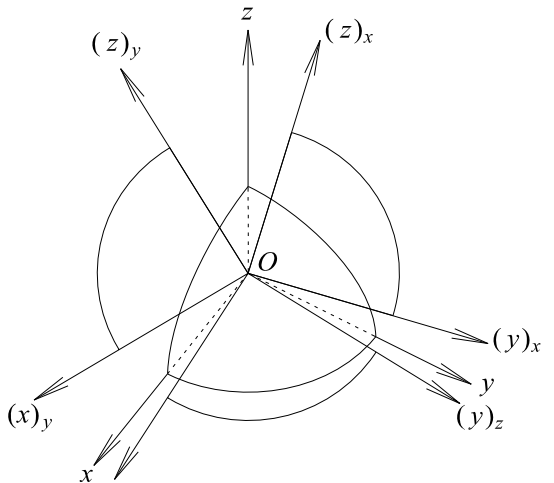

$(x)_{z}$

Fig. 6 Main related views of unit figures.

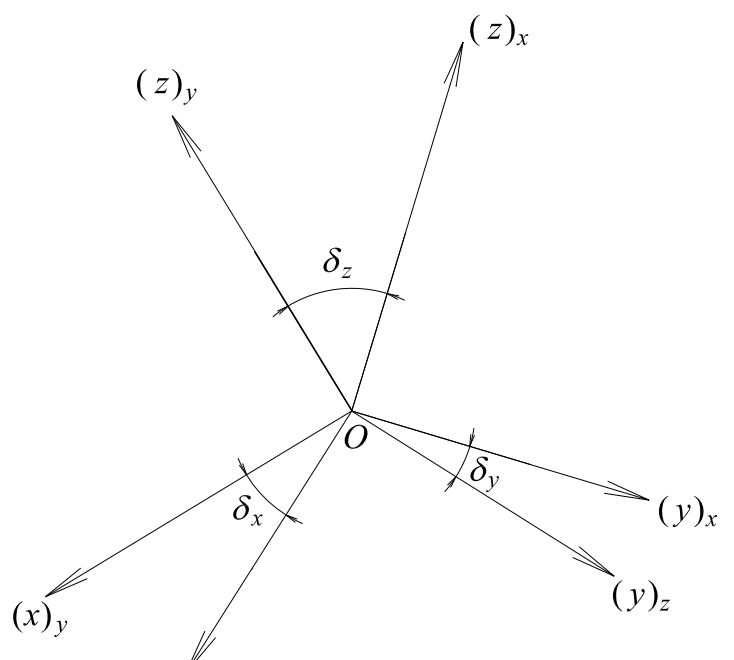

$(x)_{z}$

Fig. 7 Main related views position.

The following parallel lines are drawn: $\overline{(I)_{y}(I)_{z}}$ and $\overline{(J)_{z}(K)_{y}} ; \overline{(J)_{z}(J)_{x}}$ and $\overline{(K)_{x}(I)_{z}} ; \overline{(K)_{x}(K)_{y}}$ and $\overline{(I)_{y}(J)_{x}}$ (its demonstration is elemental).

The intersection points between those lines are called $U_{z}, U_{y}$ and $U_{z}$.

As it is shown in Fig. 8, four similar triangles have been formed $\Delta U_{x} U_{y} U_{z}, \Delta(I)_{y}(I)_{z} U_{x}, \Delta(J)_{z}(J)_{x} U_{y}$ and $\Delta(K)_{x}(K)_{y} U_{z}$ as their angles are the same.

From Fig. 9 it is deduced that $O, I, J$ and $K$ points are counterparts and they are associated to the four triangles aforementioned.

These four points form the axonometric perspective with its angles and scales. The perspective is represented by lines $x, y$ and $z$ that meet in vertex $O$. $(x)_{y}$

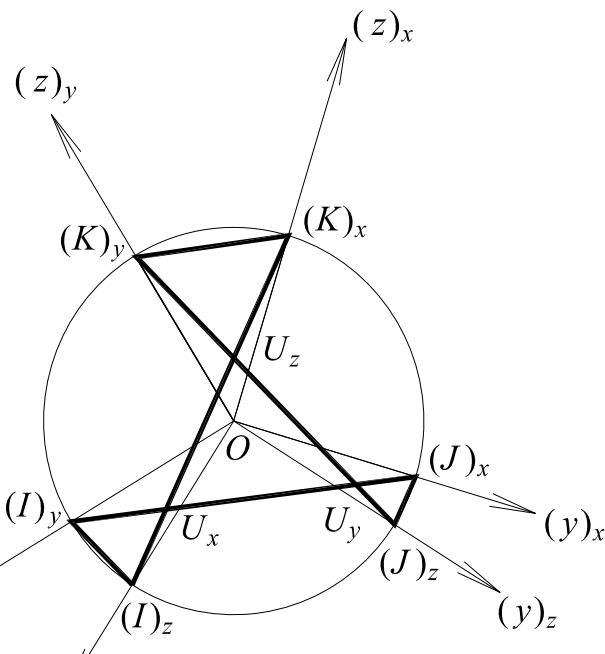

$(x)_{z}$

Fig. 8 Relationship between main related views.

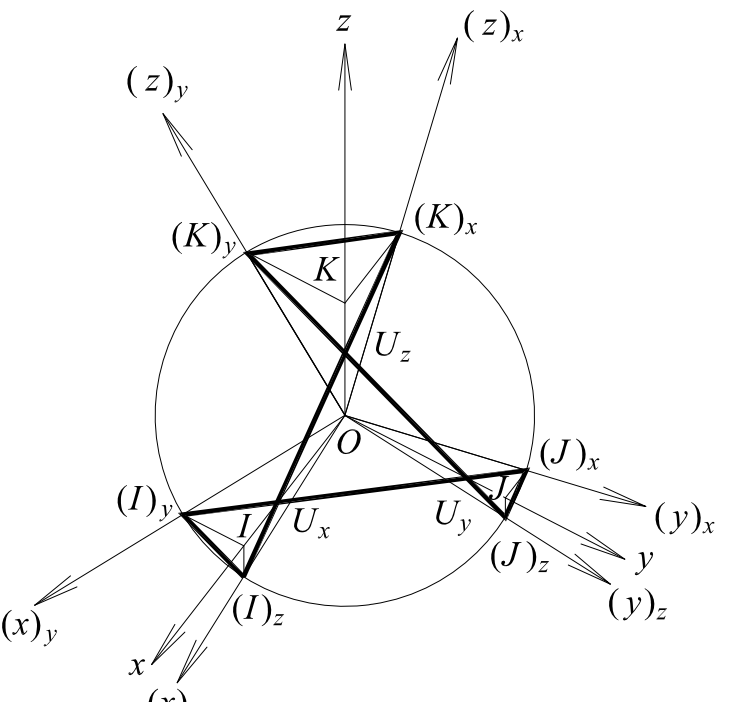

$(x)_{z}$

Fig. 9 The axonometric construction from the main related views. 
It is also possible to appreciate the similarity between Fig. 3 Trihedral system planes tilted on the main chart plane and Fig. 9 The axonometric construction from the main related views.

By making the correct operations on the Eq. (19) we can get angles and axonometric axes' scales from positional values of the main related views $\delta_{x}, \delta_{y}$ and $\delta_{z}$.

Table 1 shows angles formed between the axonometric axes from the arrangement of the quadrants of the reference trihedral planes titled down on the chart plane.

Table 1 expresses the angles in centesimal degrees.

From these angles it is possible to get other needed axonometric values in a graphical or mathematical way, following the methods described in the article.

Table 1 Axonometric angles from the positional angles of the main related views.

\begin{tabular}{|l|l|l|l|l|l|l|}
\hline & $\delta_{x}$ & $\delta_{y}$ & $\delta_{z}$ & $\alpha_{x}$ & $\alpha_{y}$ & $\alpha_{z}$ \\
\hline Isometric & $30^{\circ}$ & $30^{\circ}$ & $30^{\circ}$ & $120^{\circ}$ & $120^{\circ}$ & $120^{\circ}$ \\
\hline \multirow{5}{*}{ Dimetric } & $25^{\circ}$ & $25^{\circ}$ & $40^{\circ}$ & $125.78^{\circ}$ & $125.78^{\circ}$ & $108.43^{\circ}$ \\
\cline { 2 - 7 } & $20^{\circ}$ & $20^{\circ}$ & $50^{\circ}$ & $129.76^{\circ}$ & $129.76^{\circ}$ & $100.47^{\circ}$ \\
\cline { 2 - 7 } & $15^{\circ}$ & $15^{\circ}$ & $60^{\circ}$ & $132.37^{\circ}$ & $132.37^{\circ}$ & $95.26^{\circ}$ \\
\cline { 2 - 6 } & $10^{\circ}$ & $10^{\circ}$ & $70^{\circ}$ & $133.95^{\circ}$ & $133.95^{\circ}$ & $92.11^{\circ}$ \\
\cline { 2 - 6 } & $5^{\circ}$ & $5^{\circ}$ & $80^{\circ}$ & $134.76^{\circ}$ & $134.76^{\circ}$ & $90.48^{\circ}$ \\
\hline \multirow{5}{*}{ Trimetric } & $25^{\circ}$ & $60^{\circ}$ & $5^{\circ}$ & $101.61^{\circ}$ & $92.891^{\circ}$ & $165.50^{\circ}$ \\
\cline { 2 - 7 } & $25^{\circ}$ & $55^{\circ}$ & $10^{\circ}$ & $110.98^{\circ}$ & $96.15^{\circ}$ & $152.87^{\circ}$ \\
\cline { 2 - 7 } & $25^{\circ}$ & $50^{\circ}$ & $15^{\circ}$ & $117.95^{\circ}$ & $99.80^{\circ}$ & $142.24^{\circ}$ \\
\cline { 2 - 7 } & $25^{\circ}$ & $45^{\circ}$ & $20^{\circ}$ & $122.78^{\circ}$ & $103.88^{\circ}$ & $133.34^{\circ}$ \\
\cline { 2 - 7 } & $25^{\circ}$ & $35^{\circ}$ & $30^{\circ}$ & $127.22^{\circ}$ & $113.52^{\circ}$ & $119.26^{\circ}$ \\
\cline { 2 - 7 } & $65^{\circ}$ & $5^{\circ}$ & $104.53^{\circ}$ & $92.20^{\circ}$ & $163.27^{\circ}$ \\
\cline { 2 - 7 } & $20^{\circ}$ & $60^{\circ}$ & $10^{\circ}$ & $115.95^{\circ}$ & $94.67^{\circ}$ & $149.38^{\circ}$ \\
\cline { 2 - 7 } & $20^{\circ}$ & $55^{\circ}$ & $15^{\circ}$ & $124.17^{\circ}$ & $97.41^{\circ}$ & $138.42^{\circ}$ \\
\cline { 2 - 7 } & $20^{\circ}$ & $40^{\circ}$ & $30^{\circ}$ & $135.33^{\circ}$ & $107.70^{\circ}$ & $116.97^{\circ}$ \\
\cline { 2 - 7 } & $\ldots$ & $\ldots$ & $\ldots$ & $\ldots$ & $\ldots$ & $\ldots$ \\
\hline
\end{tabular}

\section{Conclusions}

Steps to get the tilt of a coordinate plane from the reference trihedral system on the main chart plane have been presented. This method has also been applied with all the coordinate planes that form the trihedral. Main related views are defined as those produced by the tilts of the three coordinate planes, outward from its trihedral, on the main chart plane. From this we can conclude that the new building operations facilitate and simplify the understanding of the axonometric system fundamentals. It has been demonstrated in this paper that from the main related views it is possible to build the axonometry where they come from. Analytical expressions of axonometric system elements are simplified because we have considered the reference trihedral immovable and the main chart plane variable. We can see the mathematic elegance of the equation [19]. Angles between axonometric axes have been obtained in this equation from the disposition of the quadrants of the reference trihedral planes tilted down on the chart plane. We consider that this new definition of the main related views allows a simplification in the understanding of the representation systems and its use is intended to be suitable for the purpose of computer programming and education.

\section{References}

[1] E. Caridad Yáñez, Axonometría como sistema de representación, Boletín Académico (E.T.S.A. de La Coruña) 18 (1994) 18-23.

[2] W. Farish, Treatise on isometrical perspective, The Transactions of the Cambridge Philosophical Society, Vol. I, 1820.

[3] J. L. Weisbach, Die monodimetrische und anisometrische, Projectionsmethode (Perspective), Polytechnische Mitteilungen von Volz und Kamarsch, Bd I, 1844.

[4] R. Schüssler, Orthogonale Axonometrie, Ein Lehrbuch zum Selbststudium, Leipzig, Teubner, 1905.

[5] ISO 5456:1996 Technical drawings - Projection methods, 1996.

[6] R. Ferrer Garces, La deformación por efecto de divergencia en la representación axonométrica. Boletín Académico (E.T.S.A. de La Coruña) 11 (2) 1989 26-29.

[7] F. Agrasar Quiroga, El valor simbólico de la axonometría, Boletín Académico (E.T.S.A. de La Coruña) 16 (2) (1992) 50-55.

[8] J. Krikke, Axonometry: A matter of perspective, IEEE Computer Graphics and Applications 20 (4) (2000) 7-11.

[9] P. Schreiber, Generalized Descriptive Geometry, Journal for Geometry and Graphics 6 (1) (2002) 37-59.

[10] Z. Sklenáriková and M. Pémová, The Pohlke-Schwarz Theorem and its Relevancy in the Didactics of Mathematics, Quaderni di Ricerca in Didattica 17 (2007) 152-164. 